Paidéia, 2005, 15(32), 409-416

\title{
CONHECIMENTO DE MULHERES SOBRE CÂNCER DE MAMA E DE COLO DO ÚTERO ${ }^{1}$
}

\author{
Nancy Capretz Batista da Silva² \\ Maria Aparecida Paiva Franco \\ Susi Lippi Marques \\ Universidade Federal de São Carlos
}

\begin{abstract}
Resumo: No Brasil, a incidência do câncer de mama e do colo do útero está altamente relacionada à falta de informação. É importante avaliar o conhecimento da população feminina sobre o assunto e, a partir disso, estabelecer estratégias para a diminuição das ocorrências. Assim, este trabalho visou avaliar o conhecimento de 294 mulheres- funcionárias de hospital, escolas e professoras- com idade entre 20 e 57 anos, sobre este tema, por meio de teste objetivo e questionário. Os dados revelaram que embora 89,11\% delas procurava adotar condutas preventivas, ainda há dúvidas sobre formas de incidência e prevenção do câncer de mama e colo do útero. Aponta-se a viabilidade e aplicabilidade do instrumento (teste objetivo) como recurso técnico para planejamento de intervenções que ampliem o conhecimento sobre a doença e mudem a atitude da população na prevenção e detecção precoce do câncer de mama e colo do útero no âmbito da Saúde Pública.
\end{abstract}

Palavras-Chave: Prevenção do Câncer; Câncer de Mama e Colo de Útero; Conhecimento sobre o Câncer, Medida em Psicologia; Intervenção em Psicologia.

\section{WOMEN’ KNOWLEDGE ABOUT BREAST CANCER AND COLON CANCER}

Abstract: In Brazil, breast cancer and colon cancer incidences are highly related to the lack of information. It is important to evaluate the knowledge that the feminine population has about this matter to establish strategies in order to decrease those events. In this context, the purpose of this study was to evaluate the knowledge that 294 women (hospitals, schools and universities functionaries, teachers and professors), aging from 20 to 57 years old, have about this subject employing an objective test and a questionnaire. Data revealed that although $89.11 \%$ of them tried to assume preventive conducts, there are still some doubts about breast and colon cancer incidence and prevention forms. It is supported the instrument (objective test) viability and applicability as a technical resource for a intervention planning that disseminates knowledge about the disease, which might result in a change of the population behavior on prevention and precocious detection of breast ad colon cancer in Public Health area.

Key words: Cancer Prevention; Breast Cancer and Colon Cancer; Knowledge about Cancer; Measuring in Psychology; Psychology Intervention.

Introdução: O câncer é uma das doenças mais desafiadoras desse século, devido à ausência de cura e tratamento eficaz em muitos casos e ao efeito arrebatador que causa no doente. Segundo dados do INCA (Instituto Nacional do Câncer) de 2004, ele é

\footnotetext{
1 Recebido em 13/05/2005, aceito para publicação em 24/10/2005. 2 Endereço para correspondência: Susi Lippi Marques, CECH. Departamento de Psicologia, Universidade Federal de São Carlos, Rod. Washington Luis (SP 310), Km 235, São Carlos- SP CEP: 13565905, E-mail susilmo@power.ufscar.br
}

definido como um grupo de doenças que se caracterizam pela perda do controle da divisão celular e capacidade de invadir outras estruturas orgânicas (metástase). Pivetta (2004) diz que a incidência de câncer cresce por várias razões, algumas ligadas à melhoria das condições de saúde e higiene de grandes fatias da população mundial e ao progresso da ciência, pois, os avanços no conhecimento somados a maior informação sobre a doença entre os leigos, ajudaram no diagnóstico precoce de vários tipos de 


\section{Nancy Capretz Batista da Silva}

câncer, aumentando as estatísticas de casos e mortes atribuídas a ele. Outra razão, ruim por sinal, seria o estilo de vida do homem moderno que o expõe a fatores de risco que predispõem ao câncer como fumar, tomar sol em excesso, beber demais e ter contato prolongado com produtos químicos potencialmente carcinogênicos ou vírus, além da questão genética, já que $15 \%$ das causas são hereditárias.

Em todo o mundo, 12\% das mortes são provocadas por câncer. Os anos de sobrevida aumentaram para pacientes com tumores diagnosticados e tratados em seus estágios iniciais, mas o prognóstico para os casos em que a doença já se mostra disseminada pelo organismo praticamente não se alterou.

De acordo com Pivetta (2004), no Brasil, o câncer constitui a segunda causa de morte, atrás apenas das doenças cardiovasculares. Em 2002, cerca de 400 mil novos casos foram registrados e quase 130 mil brasileiros morreram em razão da doença, representando $13,2 \%$ de todos os óbitos do país. O Ministério da Saúde estimou, em consonância com os dados de 2004 do INCA, para 2003 em todo o país, 186.155 novos casos entre os homens, levando 68.350 deles a óbito e 216.035 entre as mulheres, chegando a 58.610 óbitos.

Pivetta (2004) coloca que o câncer de mama e o de colo de útero estão entre os oito principais tipos que levam a morte no país, estando em ascenção as devidas ao câncer de mama. Por isso, é necessário conhecer um pouco mais a respeito dessas doenças que atingem grande parcela da população feminina do país, pela simples falta de informação.

O câncer de mama, provavelmente o mais temido pelas mulheres devido à alta freqüência, apresenta-se como a segunda neoplasia maligna mais incidente. A Organização Mundial da Saúde estima que, por ano, ocorram mais de 1.050 .000 casos novos deste tipo em todo o mundo (Ministério da Saúde, 2004). Ele é relativamente raro antes dos 35 anos de idade, mas acima desta faixa etária sua incidência cresce rápida e progressivamente (INCA, 2004). Por mais que as técnicas de tratamento tenham avançado, ele se constitui em um trauma psicológico para a maioria das mulheres.

De acordo com Maluf, Mori e Barros (2005), é de grande relevância que todas as pacientes diagnosticadas com câncer de mama tenham um ade- quado suporte psicológico durante todas as fases do tratamento, pois, como afirma o estudo de Moorey et al. (1989, segundo Maluf, Mori \& Barros, 2005) entre 25 e 35\% das mulheres com câncer de mama irão desenvolver ansiedade e/ou depressão em algum estágio do tratamento e que, para muitas, sem ajuda, isso torna-se incessante. Resultados encontrados em um estudo desenvolvido por Arndt, Merx, Stürmer, Stegmaier, Ziegler e Brenner (2004) indicaram que mulheres jovens com câncer de mama, em particular, mostram déficits psicológicos, e problemas financeiros. Por outro lado, fadiga incapacitante e sintomas psicológicos de depressão ou ansiedade são comumente relatadas por mulheres com câncer de mama tratado.

A importância de identificar e tratar os problemas afetivos em mulheres com câncer de mama tem sido destacada em estudos dinamarqueses em que foi verificado que pacientes com depressão tinham um risco significativamente aumentado de mortalidade (Bennett, Goldstein, Lloyd, Davenport \& Hickie, 2004). Petit e Greco (2002) afirmam que, antes da cirurgia, deveria ser oferecido suporte psicológico às pacientes, dado os efeitos psicológicos sobre a percepção de sexualidade e a própria imagem pessoal. Quando a mama, símbolo corpóreo de feminilidade, maternidade e sensualidade, é danificada, a auto-imagem da mulher pode se alterar, acarretando sentimentos de inferioridade e medo de rejeição (Gimenes, Moraes, Yamaguchi, Wanderley \& Carvalho, 1994).

Cabe salientar que sobreviventes ao câncer de mama representam o maior grupo entre os de longotermo, perfazendo aproximadamente $40 \%$ do total de sobreviventes de câncer feminino nos países desenvolvidos (Arndt \& cols, 2004).

Por outro lado, no Brasil, o câncer de mama é o que mais causa mortes entre as mulheres. Em 2003, foram estimadas 9.335 óbitos decorrentes deste tipo de câncer, sendo o segundo mais incidente entre a população feminina. Um dos fatores que contribuem para esta alta mortalidade é o avançado estágio da doença no momento em que as mulheres são submetidas ao primeiro tratamento.

Em um estudo de Ciatto, Guido e Marco (2004), a detecção precoce do câncer de mama, na fase sem sintomas, foi associada com uma média de antecipação diagnóstica de 2.6 anos comparada ao diagnósti- 
co na presença dos sintomas. As formas mais eficazes para detecção precoce do câncer de mama, de acordo com o INCA, são o exame clínico da mama e a mamografia. $\mathrm{O}$ auto-exame das mamas também pode ser realizado, mas não desobriga a mulher de se submeter ao exame clínico, que deve ser realizado anualmente. A mamografia (mastografia ou senografia) é a radiografia da mama que permite a detecção precoce do câncer, por ser capaz de mostrar lesões em fase inicial, muito pequenas. O INCA (2004) não estimula o auto-exame das mamas como estratégia isolada. Um estudo de Molina, Dalben e De Luca (2003) mostrou que a prevalência e a mortalidade da doença são maiores nas mulheres idosas, que apresentaram taxa menor de oportunidades de diagnóstico precoce, por serem menos informadas sobre a periodicidade correta da auto-palpação e terem menor freqüência de mamografia e exame clínico das mamas do que as mais jovens. O grau de escolaridade também mostrou significância estatística; as mulheres com mais anos de estudo teriam melhores oportunidades de informação. Mas, qualquer mulher pode vir a ter um câncer de mama. No entanto, há determinados grupos com maiores probabilidades.

Segundo o INCA (2004), a história familiar é um importante fator de risco para o câncer de mama, especialmente se mãe e irmã foram acometidas na pré-menopausa, o que as colocaria como tendo uma história familiar de primeiro grau, e, então, cerca de duas vezes mais risco de desenvolver câncer de mama; $10 \%$ dos casos de câncer de mama achamse ligados a uma história familiar deste câncer. Além disso, as mulheres que já tiveram a doença em uma das mamas possuem maiores probabilidades de desenvolver câncer na outra. A idade constitui também fator importante de risco, havendo um aumento rápido da incidência com o passar dos anos. A menarca precoce, a menopausa tardia (instalada após os 50 anos de idade), a ocorrência da primeira gravidez após os 30 anos e a nuliparidade constituem também fatores de risco para o câncer de mama. Ainda é controvertida a associação do uso de contraceptivos orais com o aumento do risco para certos subgrupos de mulheres como as que utilizaram dosagens elevadas de estrogênio, ou que ingeriram a medicação por longo período, ainda as que inciaram a tomada de anticoncepcional em idade precoce, antes da primeira gravidez. Por outro lado, a ingestão regular de álcool, mesmo que em quantidade moderada, é identificada como fator de risco, assim como a exposição a radiações ionizantes em idade inferior a 35 anos. Estudos têm sugerido que a prevenção do tabagismo, alcoolismo, obesidade e sedentarismo reduzem o risco de câncer de mama (INCA, 2004).

O câncer do colo do útero, explica o INCA (2004), ainda é relevante, em termos de casos novos e de morte no mundo, principalmente por conta das estatísticas referentes aos países em desenvolvimento - $80 \%$ dos casos novos. Entretanto, mesmo assim, no Brasil e em outros países de seu nível, a magnitude das taxas de mortalidade são bem menores que as de incidência; estima-se que aqui o câncer do colo do útero seja o terceiro mais comum na população feminina, superado pelo de pele e de mama; ele representa $10 \%$ de todos os tumores malignos em mulheres, estimando-se em 4.110 o número de óbitos para o ano de 2003. Similarmente ao que ocorre com o câncer de mama, o estágio da doença já é avançado quando detectada.

O INCA (2004) afirma que os fatores sociais, ambientais e hábitos de vida, tais como baixas condições sócio-econômicas, atividade sexual antes dos 18 anos de idade, pluralidade de parceiros sexuais, vício de fumar (diretamente relacionado à quantidade de cigarros), parcos hábitos de higiene e uso prolongado de contraceptivos orais são os principais fatores de risco identificados para o câncer do colo do útero. Além disso, a presença do HPV é confirmada em 99\% dos casos; o comentário é de que o HPV exerce papel importante no desenvolvimento da displasia das células cervicais e na sua transformação em cancerosas (Coelho, Prado, Pereira Sobrinho, Hamada, Landman, Pinto, Nonogaki \& Villa, 2004). Resultados de uma pesquisa de Maciag e Villa (1999) mostraram que a bagagem genética é importante para definir susceptibilidade maior ou menor a infecção pelo HPV, o maior fator de risco para o desenvolvimento de lesões malignas no colo do útero.

Até o presente momento, as mulheres com tumores do colo de útero são a parcela da população mais atingida pelo HPV de alto risco oncogênico. Então, torna-se importante realizar campanhas para a conscientização da necessidade de exames ginecológicos preventivos, pois a detecção precoce da le- 


\section{Nancy Capretz Batista da Silva}

são causada pelo HPV permite a utilização de abordagens terapêuticas menos invasivas, ao contrário do que ocorre quando são detectados tumores de grau avançado. Como são os homens que transmitem o vírus para as mulheres, eles também devem ser alvo da educação preventiva (Souto, Falhari \& Cruz, 2005).

Nos países desenvolvidos, o aumento da incidência de casos de câncer de mama e colo do útero, acompanhado pela redução da mortalidade está associado à detecção precoce e à oferta de tratamento adequado. No Brasil existe retardamento do diagnóstico e nem sempre a terapêutica é adequada (Ministério da Saúde, 2004).

Tendo-se em vista o impacto que o câncer pode ocasionar, estratégias profiláticas, tanto em nível da saúde mental quanto física, são de fundamental importância para a diminuição das ocorrências e melhoria do lidar com a doença. O conhecimento de formas efetivas de prevenção e manejo de condições sócio-ambientais são possíveis e isto deve ser assu- mido pelos profissionais de saúde de modo a elevar a qualidade de vida das pessoas.

A partir desses dados, este trabalho teve por objetivo, avaliar o conhecimento de mulheres sobre o câncer de mama e do colo do útero por meio de um teste objetivo e questionário preliminar, visando checar a adequabilidade destes enquanto recurso técnico auxiliar no planejamento de programas de intervenção.

\section{Método \\ Participantes}

Este estudo contou com uma amostra de 294 mulheres, com idade entre 20 e 57 anos, sendo 64 funcionárias de hospitais (FH), 146 professoras (P) e 84 funcionárias de escolas ou universidades (FE). Todas as colaboradoras eram moradoras da cidade de São Carlos - SP e seu nível de escolaridade variou de primeiro grau incompleto a terceiro grau completo, conforme apresentado na Tabela 1.

Tabela 1: Caracterização das participantes por nível de escolaridade.

\begin{tabular}{|c|c|c|c|c|c|c|c|}
\hline \multirow{2}{*}{\multicolumn{2}{|c|}{$\begin{array}{c}\text { VARIÁVEIS } \\
\text { CARACTERIZADORAS }\end{array}$}} & \multicolumn{6}{|c|}{ ESCOLARIDADE } \\
\hline & & $\begin{array}{c}1^{\circ} \mathrm{Grau} \\
\text { incompleto }\end{array}$ & $\begin{array}{c}1^{\circ} \text { Grau } \\
\text { completo }\end{array}$ & $\begin{array}{c}2^{\circ} \mathrm{Grau} \\
\text { incompleto }\end{array}$ & $\begin{array}{c}2^{\circ} \text { Grau } \\
\text { completo }\end{array}$ & $\begin{array}{c}3^{\circ} \mathrm{Grau} \\
\text { incompleto }\end{array}$ & $\begin{array}{c}3^{\circ} \mathrm{Grau} \\
\text { completo }\end{array}$ \\
\hline \multirow{4}{*}{ 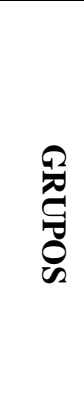 } & $\begin{array}{l}\text { Funcionários de } \\
\text { Hospitais }(\mathrm{FH})\end{array}$ & 3 & 7 & 5 & 25 & 13 & 11 \\
\hline & Professoras (P) & 0 & 0 & $2 *$ & 11 & 10 & 123 \\
\hline & $\begin{array}{c}\text { Funcionárias de } \\
\text { Escolas ou } \\
\text { Universidades (FE) }\end{array}$ & 2 & 4 & 11 & 22 & 6 & 39 \\
\hline & Total & 5 & 11 & 18 & 58 & 29 & 173 \\
\hline
\end{tabular}

*Estagiárias de Magistério.

\section{Material}

Foi utilizado como instrumento um teste objetivo, composto por 22 itens de múltipla escolha sobre conhecimentos a respeito de câncer de mama e de colo do útero, que foi construído e testado em uma amostra piloto em estudo anterior (Correia \& Marques, 2002), tendo as análises dos itens evidenciado índices psicométricos satisfatórios o de dificuldade dos itens variou entre 0,32 e 1,00, a Discriminabilidade de
$30 \%$ e $66 \%$ e a Precisão, obtida através da técnica das duas metades, corrigida pela fórmula de Spearman-Brown, foi de 0,50. A partir deste estudo inicial, imprescindível no processo de proposição de instrumentos de medida, o teste foi confirmado como adequado para aplicação tanto em relação ao conteúdo de seus itens quanto à clareza de entendimento dos mesmos.

Juntamente com o instrumento, as participan- 
tes recebiam uma folha de rosto com instruções e esclarecimentos para o preenchimento do teste e, para caracterização da amostra, um questionário de dados pessoais e informações sobre casos de câncer na família, contato desta com pessoas com câncer, tipo de prevenção adotada frente à doença, freqüência de consultas ao ginecologista e formas de tratamento conhecidas.

\section{Procedimento}

A aplicação do instrumento e do questionário, na amostra de 294 colaboradoras, ocorreu após a obtenção da autorização dos responsáveis dos diferentes setores dos hospitais e universidades, assim como dos diretores de escolas, quando a aplicação foi realizada na própria instituição ${ }^{3}$. Após a autorização dos responsáveis, as funcionárias foram contatadas e, mediante a exposição dos objetivos do estudo, aderiram ou não à pesquisa, sendo a resposta ao instrumento e ao questionário realizada nos intervalos do expediente de trabalho. Nos casos de preferência por responder fora do local do trabalho, foram agendados dia, horário e local de acordo com a possibilidade dos participantes.

\section{Resultados}

Os dados do questionário preliminar passaram por análises qualitativa e quantitativa. Foi feita uma comparação dos grupos amostrais para os escores obtidos no teste e análise dos itens do teste, por temática, segundo os acertos gerais e grupais.

Caracterização das participantes: $169 \mathrm{mu}-$ lheres eram casadas [35 (FH), 86 (P) e 48 (FE)] 57,48\%; 1 era amasiada (FE) - 0,003\%; 96 solteiras [23 (FH), 48 (P) e 25 (FE)] - 32,65\%, 11 divorciadas [2 (FH), 5 (P) e 4 (FE)] - 0,037\%, 5 desquitadas [2 (FH), 2 (P) e 1 (FE) $-0,017 \%, 4$ separadas [1 (P) e 3 (FE) $-0,014 \%$ - e 3 viúvas [(1 (FH) e $2(\mathrm{P})]$ -

\footnotetext{
${ }^{3}$ Este estudo contou com a participação voluntária de mulheres que, após terem sido esclarecidas quanto ao objetivo da pesquisa, tinham liberdade para aderirem ou não ao estudo. As participantes foram informadas sobre a possibilidade de interromper a aplicação caso desejassem e seus dados não seriam considerados no estudo. Foi assegurado às colaboradoras o anonimato por ocasião da publicação dos resultados. Todos os cuidados éticos foram adotados visando a integridade e bem-estar das participantes, conforme estabelecido pela resolução 196/96 do Ministério da Saúde.
}

$0,01 \%$. As 4 restantes não preencheram esta questão [1 (FH), 2 (P) e 1 (FE)].

Foi constatado que das 294 colaboradoras 185 (62,92\%) tinham caso de câncer na família [40 (FH), 97 (P) e 48 (FE)], sendo que desses, 34,59\% era câncer de mama ou de colo do útero; 182 (61,9\%) declararam ter tido contato com portadoras de câncer de mama, 58 (19,73\%) de colo do útero e 173 (58,84\%) com portadores de outro tipo de câncer.

A maioria delas, 262 ou 89,11\% [85,94\% (FH), $96,57 \%$ (P) e $78,57 \%$ das (FE)], procurava se prevenir contra o câncer, fazendo exames periódicos, acompanhamento médico, procurando alimentação adequada, evitando exposição solar, uso de fumo e praticando exercícios físicos. Grande parte da amostra, 275 $(93,54 \%)$, costuma ir ao ginecologista $[93,75 \%(\mathrm{FH})$, 95,89\% (P), 89,58\% (FE)],com freqüência semestral $(30,54 \%)$ e anual (60\%).

\section{Análise comparativa dos grupos}

O teste de Kruskal-Wallis foi utilizado para ver as diferenças entre as médias dos escores obtidos nos grupos analisados $(\mathrm{N}=294)$. A estatística de Kruskal-Wallis teve distribuição $X^{2}=16,0332$ com (2 $\mathrm{gl}), \mathrm{p}=0,0003$, mostrando diferença significativa entre o conhecimento dos grupos. Comparações revelaram que não há diferença entre os escores obtidos no teste para o grupo das funcionárias de hospital e professoras $\left[\mathbf{F H} \times \mathbf{P}=X^{2}=3,9571 ; \mathrm{gl}=1(\mathrm{p}=0,046)\right]$ e entre funcionárias de escola e professoras $\left[\mathbf{F E} \times \mathbf{P}=X^{2}=\right.$ 9,4585; $g \mathrm{l}=1 ;(p=0,0021)]$. Contudo, houve diferença significativa nas respostas das funcionárias de hospital e de escola $\left[\mathbf{F H} \times \mathbf{F E}=X^{2}=11,856 ; \mathrm{gl}=1\right.$ $(p=0,00057)]$. Na Tabela 2 encontram-se os escores gerais do teste para a amostra dividida em seus grupos de participantes.

Vabela 2: Amplitude dos escores e escore médio para os grupos de participantes e geral.

\begin{tabular}{c|c|c}
\hline GRUPOS & $\begin{array}{c}\text { AMPLITUDE } \\
\text { DOS ESCORES }\end{array}$ & $\begin{array}{c}\text { ESCORE } \\
\text { MÉDIO }\end{array}$ \\
\hline FH & $5-22$ & 15,13 \\
\hline P & $5-21$ & 14,61 \\
\hline FE & $4-20$ & 13,46 \\
\hline GERAL & $4-22$ & 14,39 \\
\hline
\end{tabular}




\section{Nancy Capretz Batista da Silva}

\section{Análise dos itens do teste em função da sua temática}

Foi investigado o número de acertos em cada item, e a posterior análise do conteúdo de acordo com o grau de dificuldade que apresentaram ao grupo de participantes.

Dos vinte e dois itens que compunham o instrumento, 14 referiam-se ao câncer de mama e 8 ao de colo de útero. O número de acertos das participantes por itens acham-se na Tabela 3.

Tabela 3: Acertos das participantes por itens em porcentagem por grupo e geral.

\begin{tabular}{c|c|c|c|c}
\hline \multirow{2}{*}{ ITENS } & \multicolumn{3}{|c|}{ GRUPOS } & GERAL \\
\cline { 2 - 4 } & $\begin{array}{c}\mathrm{FH} \\
(\mathrm{N}=64)\end{array}$ & $\begin{array}{c}\mathrm{P} \\
(\mathrm{N}=146)\end{array}$ & $\begin{array}{c}\mathrm{FE} \\
(\mathrm{N}=84)\end{array}$ & $(\mathrm{N}=294)$ \\
\hline 1 & 9 & 11 & 17 & 12 \\
\hline 2 & 30 & 45 & 33 & 38 \\
\hline 3 & 91 & 89 & 82 & 87 \\
\hline 4 & 61 & 64 & 58 & 62 \\
\hline 5 & 80 & 82 & 65 & 77 \\
\hline 6 & 72 & 88 & 80 & 82 \\
\hline 7 & 72 & 67 & 63 & 67 \\
\hline 8 & 73 & 71 & 57 & 68 \\
\hline 9 & 53 & 35 & 31 & 38 \\
\hline 10 & 20 & 35 & 35 & 32 \\
\hline 11 & 95 & 93 & 94 & 94 \\
\hline 12 & 83 & 76 & 65 & 74 \\
\hline 13 & 75 & 66 & 67 & 68 \\
\hline 14 & 89 & 77 & 76 & 80 \\
\hline 15 & 89 & 84 & 74 & 82 \\
\hline 16 & 77 & 50 & 55 & 57 \\
\hline 17 & 70 & 68 & 61 & 66 \\
\hline 18 & 55 & 44 & 50 & 48 \\
\hline 19 & 78 & 80 & 68 & 76 \\
\hline 20 & 89 & 88 & 79 & 86 \\
\hline 21 & 70 & 70 & 56 & 66 \\
\hline 22 & 81 & 77 & 81 & 79 \\
\hline & & & & \\
\hline
\end{tabular}

A análise pontual dos itens, da amostra como um todo, revelou que o 1 , apresentou apenas 12,24\% de acerto, sendo considerado um item difícil. Ele tratou das formas de prevenção do câncer de mama e o resultado parece indicar que a população investigada não conhece ou apresenta dúvidas sobre este tema. Porém, sua resposta correta envolvia a discussão do papel da pílula contraceptiva, algo ainda contraditório no meio científico, evidenciando a necessidade de atualização quanto às descobertas mais recentes. Os itens 2 (definição do câncer), 9 (hormônio que contribui para o aparecimento do câncer mamário) e 10 (qual o cancer de mama que atinge sua maior probabilidade de incidência), tiveram acerto de 38,09\%, $37,75 \%$ e $31,63 \%$, respectivamente, portanto maior do que do item 1 , mas ainda considerados difíceis, indicando um baixo conhecimento das participantes sobre estes tópicos. Os itens 16 ( principal via de contágio do câncer de colo de útero) e 18 (melhor época para fazer exame ginecológico, para detectar câncer de colo de útero) tiveram porcentagens de acerto de 57,14\% e 47,95\%, respectivamente, portanto, uma dificuldade média, mostrando que ainda existem dúvidas quanto a estes temas. Os itens 4 (tratamento do câncer de mama), 5 (presença de sintomas no estágio inicial do câncer de mama), 7(formas de tratamento, 8 (incidência de câncer de mama em pessoas do sexo masculino), 12 (cura), 13 (indícios de câncer de mama), 14 (exame de mamografia), 17 (população que deve fazer exame para prevenção do câncer do colo de útero), 19 (fatores de risco para o câncer do colo uterino), 21(cuidados a serem tomados antes do exame de câncer de colo de útero) e 22 tiveram (faixa etária em que as mulheres são mais atingidas pelo câncer do colo de útero) tiveram $61,9 \%, 76,87 \%, 67 \%, 67,68 \%, 74,48 \%, 68,36 \%, 79,59 \%$, $66,32 \%, 76,19 \%, 65,98 \%$ e $78,91 \%$, respectivamente, de acertos, indicando serem fáceis, evidenciando o razoável conhecimento da população investigada sobre estes tópicos. Os itens 3 (métodos capazes de diagnosticar o câncer de mama), 6 (especialidades médicas que tratam ou diagnosticam o câncer de mama), 11 (conjunto de hábitos que ajuda na prevenção do câncer de mama, 15 (vírus presente na maioria dos casos e exame mais eficaz para detectá-lo) e 20 (sintomas relacionados com o câncer de colo de útero) tiveram acerto de 87,41\%, $92,31 \%, 93,87 \%, 81,97 \%$ e $85,71 \%$, respectivamente, indicando serem itens bem fáceis, mostrando temas sobre os quais a população estava atualizada.

\section{Discussão}

Os resultados permitem reflexões importantes e podem contribuir para melhor compreensão a respeito do nível de conhecimento e efetiva prevenção do câncer de mama e colo do útero. Alguns estudos (Molina \& cols, 2003) apontam como fator significante o fato de que mulheres com mais anos de estudo teriam melhores oportunidades de diagnóstico precoce do câncer de mama. Os dados deste estudo, de certa maneira suportam esta proposição, já que a maioria 
da população investigada tem alto nível de escolaridade e atingiu índices de acerto acima de 80\% nos itens referentes aos métodos de diagnóstico, especialidades médicas e conjunto de hábitos que ajuda na prevenção do câncer de mama.

Por outro lado, tomando-se o desempenho da amostra como um todo, percebe-se que a população investigada não conhece de maneira satisfatória ou apresenta dúvidas sobre alguns pontos. Apoiando-se no nível de escolaridade das participantes, em sua maioria (88\%) possuindo de $2^{\circ}$. grau completo a $3^{\circ}$. grau completo, esperar-se-ia um melhor escore geral no teste de acordo com a proposição de Molina e cols (2003). O índice de 65\% de acerto (escore médio de 14,394) não parece adequado para a manutenção de um sistema de prevenção e saúde da população.

Em relação aos grupos de mulheres investigados - funcionárias de hospitais, professoras e funcionárias de escolas ou universidades - pode-se verificar que a amplitude dos escores obtidos pelas participantes no teste foi de 15,125 (FH); 14,609 (P) e 13,464 (FE) correspondendo às porcentagens de $69 \%$, $66 \%$ e $61 \%$ de acertos, respectivamente. Estes dados, de certa maneira, aumentam a preocupação quanto à desinformação da população investigada. Esperava-se que funcionárias de hospitais, que têm maior contato com o tema, tratando de pacientes com a doença, acompanhando/fazendo diagnósticos, entre outras atividades, deveriam apresentar escores de acerto mais elevados. Da mesma maneira, as professoras, veículo potencial de transmissão de informação e conhecimento, também poderiam estar mais atualizadas quanto a informações sobre saúde.

Ainda em função da amplitude dos escores obtidos pode ser notado que algumas participantes, independente do grupo, mostraram uma ausência preocupante de conhecimento sobre vários aspectos das doenças, o que pode estar relacionado ao diagnóstico tardio, enquanto que a importância do diagnóstico precoce é ressaltada (Ciatto \& cols., 2004; INCA, 2004; Molina \& cols., 2003; Souto \& cols., 2005; Ministério da Saúde, 2004) e tem maior probabilidade de ocorrer se houver conhecimento prévio do assunto.

Outro dado que chama a atenção e merece reflexão reside no fato de que a maioria das participantes (N=275 - 93,54\%) indicou possuir o hábito de realizar consultas periódicas com o médico ginecolo- gista; infere-se, então, que as participantes ou não estão compreendendo a linguagem adotada pelo médico ou dando atenção às informações fornecidas na consulta ou, ainda, que estas (consultas) são carentes de diálogos sobre prevenção e fatores de risco para desenvolvimento da doença.

\section{Considerações finais}

As incidências de câncer de mama e de colo de útero continuam elevadas no nosso país (Ministério da Saúde, 2004) e as taxas de mortalidade precisariam ser reduzidas. Assim, a partir dos dados deste estudo que mostram a necessidade de melhoria na informação sobre o assunto, projetos de intervenção podem ser pensados, para levar ao conhecimento das condutas preventivas, incluindo uma revisão dos programas de disseminação de informações e prevenção existentes que possam resultar em mudança de atitude da população.

Neste sentido, aponta-se a importância de intervenções fortemente direcionadas à prevenção, através de ações educativas. Associado a isto se faz necessário a disponibilidade recursos diagnósticos e tratamento oportuno, destacando-se o psicológico tem sido ressaltado diante da associação constatada entre o câncer, principalmente o câncer de mama, e sintomas psicológicos (Maluf \& cols., 2005; Arndt \& cols., 2004; Bennett \& cols., 2004; Petit \& Greco, 2002; Gimenes \& cols., 1994).

Quanto ao instrumento de avaliação utilizado, em termos psicométricos, ele revelou ser um recurso técnico de medida valioso a ser utilizado em programas de intervenção, sendo preciso apenas pequenos ajustes quanto a algumas terminologias, para se adequar à possibilidade da testagem em uma amostra expandida, e com diferentes níveis de escolaridade ${ }^{4}$.

\footnotetext{
${ }^{4}$ Embora o instrumento tenha passado, na fase de elaboração, pela análise teórica com peritos na área de conhecimento (Análise de Conteúdo) e por pessoas da amostra estudada (Análise Semântica) alguns termos e expressões mostraram-se de certa forma confusos como, por exemplo, "via de contágio” na questão 16. Outras terminologias ainda são um tanto desconhecidas pela população em geral (mastologia, menarca, vírus HPV). Neste caso, podemos em algumas situações sugerir a alteração da palavra (menarca para primeira menstruação), mas em outras se torna notória a falta de informação (vírus HPV). Sendo assim, os aspectos semânticos acima citados (menarca e via de contágio) devem ser considerados pelo profissional que estará utilizando o instrumento, o qual deverá levar em consideração as características da amostra (nível instrucional) na qual o mesmo será administrado.
} 
416 Nancy Capretz Batista da Silva

A presente investigação evidenciou, portanto, que se deve atentar para a necessidade de elaboração e implementação de estratégias que envolvam e mobilizem a população pensando nas questões ligadas à Saúde Pública. E a viabilidade e aplicabilidade do instrumento proposto, que fornece dados objetivos sobre o conhecimento da população alvo de programas a respeito do tema tratado, oferece uma contribuição metodológica ao seu estudo.

\section{Referências Bibliográficas}

Arndt, V., Merx, H., Stürmer, T., Stegmaier, C., Ziegler, H. \& Brenner, H. (2004). Age-specific detriments to quality of life among breast cancer patients one year after diagnosis. European Journal of Cancer, 40 (5), 673-680.

Bennett, B., Goldstein, D., Lloyd, A., Davenport, T. \& Hickie, I. (2004). Fatigue and psychological distress - exploring the relationship in women treated for breast cancer. European Journal of Cancer, 40 (11), 1689-1695.

Ciatto, S., Guido, M. \& Marco, Z. (2004). Prognostic impact of the early detection of metachronous contralateral breast cancer. European Journal of Cancer, 40 (10), 1496-1501.

Coelho, F. R. G., Prado, J. C. M., Pereira Sobrinho, J. S., Hamada, G., Landman, G., Pinto, C. A., Nonogaki, S. \& Villa, L. L. (2004). Estrogen and progesterone receptors in human papilloma virus-related cervical neoplasia. Brazilian Journal of Medical and Biological Research, 37 (1), 83-88.

Correia, L. L. \& Marques, S. L. (2002). Instrumento para avaliação em serviços de intervenção em psicologia na prevenção do câncer de mama e colo do útero [Resumo]. Resumos de Comunicação Científica da XXXII Reunião Anual de Psicologia, 420.

Gimenes, M. G. G., Moraes, M. C., Yamaguchi, N. H., Wanderley, K. S. \& Carvalho, M. M. J. (1994). Introdução a Psiconcologia. São Paulo: Editorial Psy.

Instituto Nacional do Câncer - INCA. Câncer. Retirado do world wide web em 30/04/2004: http:// www.inca.gov.br.
Maciag, P. C. \& Villa, L. L. (1999). Genetic susceptibility to HPV infection and cervical cancer. Brazilian Journal of Medical and Biological Research, 32 (7), 915-922.

Maluf, M. F. M.; Mori, L. J. \& Barros, A. C. S. D. (2005). O impacto psicológico do câncer de mama. Revista Brasileira de Cancerologia, 51(2), 149-154.

Ministério da Saúde. (2004). Controle do Câncer de Mama - Documento de Consenso. Revista Brasileira de Cancerologia, 50 (2), 77-90.

Molina, L., Dalben, I. \& De Luca, L. A. (2003). Análise das oportunidades de diagnóstico precoce para as neoplasias malignas de mama. Revista da Associação Médica Brasileira, 49 (2), 185-190.

Petit, J.Y., Greco, M., (2002). Quality control in prophylactic mastectomy for women at high risk of breast cancer. European Journal of Cancer, 38 (1), 23-26.

Pivetta, M. (2004). Câncer, esperanças divididas. Pesquisa FAPESP, 99, 46-53.

Souto, R.; Falhari, J. P. B.; \& Cruz, A. D. (2005). O Papilomavírus humano: um fator relacionado com a formação de neoplasias. Revista Brasileira de Cancerologia, 51(2), 155-160. 